\title{
Moving Steganography and Steganalysis from the Laboratory into the Real World
}

\author{
Andrew D. Ker \\ Dept. of Computer Science \\ University of Oxford \\ Oxford OX1 3QD, UK \\ adk@cs.ox.ac.uk \\ Rémi Cogranne \\ LM2S - UMR STMR CNRS \\ Troyes Univ. of Technology \\ 10004 Troyes, France \\ remi.cogranne@utt.fr
}

\author{
Patrick Bas \\ LAGIS CNRS \\ Ecole Centrale de Lille \\ 59651 Villeneuve d'Ascq, FR \\ patrick.bas@ec-lille.fr \\ Scott Craver \\ Dept. of ECE \\ Binghamton University \\ Binghamton, NY 13902 \\ scraver@binghamton.edu
}

\author{
Rainer Böhme \\ University of Münster \\ Leonardo-Campus 3 \\ 48149 Münster, Germany \\ rainer.boehme@wwu.de \\ Tomáš Filler \\ Digimarc Corporation \\ 9405 SW Gemini Drive \\ Beaverton, OR 97008 \\ tomas.filler@digimarc.com
}

\author{
Jessica Fridrich \\ Dept. of ECE \\ Binghamton University \\ Binghamton, NY 13902 \\ fridrich@binghamton.edu
}

\author{
Tomáš Pevný \\ Agent Technology Group \\ CTU in Prague \\ Prague 16627, Czech Rep. \\ pevnak@gmail.com
}

\begin{abstract}
There has been an explosion of academic literature on steganography and steganalysis in the past two decades. With a few exceptions, such papers address abstractions of the hiding and detection problems, which arguably have become disconnected from the real world. Most published results, including by the authors of this paper, apply "in laboratory conditions" and some are heavily hedged by assumptions and caveats; significant challenges remain unsolved in order to implement good steganography and steganalysis in practice. This position paper sets out some of the important questions which have been left unanswered, as well as highlighting some that have already been addressed successfully, for steganography and steganalysis to be used in the real world.
\end{abstract}

\section{Categories and Subject Descriptors}

D.2.11 [Software Engineering]: Software ArchitecturesInformation hiding; H.1.1 [Models and Principles]: Systems and Information Theory-Information theory

\section{Keywords}

Steganography; Steganalysis; Security Models; Minimal Distortion; Optimal Detection; Game Theory

Permission to make digital or hard copies of all or part of this work for personal or classroom use is granted without fee provided that copies are not made or distributed for profit or commercial advantage and that copies bear this notice and the full citation on the first page. To copy otherwise, to republish, to post on servers or to redistribute to lists, requires prior specific permission and/or a fee.

IH\&MMSec'13, June 17-19, 2013, Montpellier, France.

Copyright 2013 ACM 978-1-4503-2081-8/13/06 ...\$15.00.

\section{INTRODUCTION}

Steganography is now a fairly standard concept in computer science. One occasionally reads, in mainstream media, of criminals hiding information in digital media $([1,4]$, see $[3]$ for other links) and, recently, of malware using it to conceal communications with command and control servers [5]. In the 1990s, the possibility of digital steganography served as an argument in debates about regulating cryptography, and it allegedly convinced some European governments to liberalize the use of cryptography [31]. We also read of the desire for certain privacy-enhancing technologies to use steganography to evade censorship [67]. If steganography becomes commonly used, so should steganalysis, though the concept is not as well recognized in nonspecialist circles.

However, where details of real-world use of steganography are known, it is apparent that they bear little resemblance to techniques described in modern literature. Indeed, they often suffer from flaws known to researchers for more than a decade. How has practice become so disconnected from research? The situation is even more stark in steganalysis, where most researchers would agree that their detectors work well only in laboratory conditions: unlike steganography, even if practitioners wanted and were technically able to implement state-of-the-art detectors, their accuracy would be uneven and unreliable.

The starting point for scientific research is to make a model of the problem. The real world is a messy place, and the model is an abstraction which removes ambiguities, sets certain parameters, and makes the problem amenable to mathematical analysis or empirical study. In this paper we contend that knowledge is the most important component in a model of the steganography and steganalysis problems. Does the steganographer have perfect knowledge about their source of covers? Does the steganalyst know the embedding method used by the steganographer? There are many questions of this type, often left implicit in early research. 
By considering different levels of knowledge, we identify a number of models of the steganography and steganalysis problems. Some of them have been well-studied but, naturally enough, it is usually the simplest models which have received the most attention. Simple models may (or may not) provide robust theoretical results giving lower or upper bounds, and they increase our understanding of the fundamental problems, but they are tied to the laboratory. In this paper we identify the models which bring both steganography and steganalysis nearer to the real world. In many cases the scientific community has barely scratched their surface, and we highlight open problems which are, in the view of the authors, important to address in future research.

At the present time, steganography and steganalysis research divides into two cover types: digital media (primarily compressed and uncompressed images, but also video and audio) and network traffic (timing channels and the content of web traffic). The authors of this paper have their interest mainly in the former, and we contend that steganography and steganalysis is significantly more sophisticated in this domain than in network channels. Although network-based steganography is perhaps closer to real-world implementation, we will argue that the field needs to learn lessons from digital media steganography.

Many of the principles in this paper apply to any type of cover, but we shall be motivated by some general properties of digital media: the complexity of the cover and the lack of perfect models, the relative ease of (visual) imperceptibility as opposed to undetectability, and large capacity per object. When, in examples, we refer to spatial domain we mean uncompressed images, and DCT or transform domain refers to JPEG-compressed images, both grayscale unless otherwise mentioned.

The paper has a simple structure. In section 2 we discuss current solutions, and open problems, relevant to applying steganography in the real world. In section 3 we do the same for steganalysis.

\section{The Steganography Problem}

We briefly recapitulate the steganography problem, refining Simmons' original Prisoners' Problem [92] to the contemporary definition of steganography against a passive warden.

A sender, often called Alice but who will throughout the paper be known as the steganographer, wishes to send a covert communication or payload to a recipient. She possesses a source of covers drawn from a larger set of possible communications, and there exists a channel for the communications (for most purposes we may as well suppose that the communication is unidirectional). The channel is monitored by an adversary, also known as an attacker or Warden but for the purposes of this paper called the steganalyst, who wishes to determine whether payload is present or not.

One solution is to use a channel that the adversary is not aware of. This is how traditional steganography has reportedly been practiced since ancient times, and most likely prevails in the Internet age [46]. Examples include tools that hide information in metadata structures, at the end of files where standard parsers ignore it [103], or modifying network packet headers such as TCP time stamps [37]. (See [74] for a systematic discussion.)

However, this approach is not satisfactory because it relies on the adversary's ignorance, a form of "security through obscurity". In Simmons' formulation, inspired by conservative assumptions typical in cryptology, the steganalyst is granted wide knowledge: the contents of the channel is perfectly observable by both parties, writable by the steganographer, and (for the "passive Warden" case which dominates this paper) read-only by the steganalyst. To enable undetectability, we must assume that cover messages run through the channel irrespective of whether hidden communication takes place or not, but this is something that we will need to make more precise later. The intended recipient of the covert payload is distinguished from the steganalyst by sharing a secret key with the steganographer (how such a key might be shared will be covered in section 2.5).

As we shall see later, this model is still imprecise: the Warden's aims, the parties' knowledge about the cover source, and even their knowledge about each others' knowledge, all create different versions of the steganography and steganalysis problems.

We fix some notation used throughout the paper. Cover objects generated by Alice's source will be denoted by $\mathbf{X}$, broken down where necessary into $n$ elements (e.g. pixels in the spatial domain pixels, or DCT coefficients in the transform domain) $X_{1}, \ldots, X_{n}$. The objects emitted by the steganographer - which may be unchanged covers or payloadcarrying stego objects - will be denoted $\mathbf{Y}$, or sometimes $\mathbf{Y}_{\beta}$ where $\beta$ denotes the size of the payload relative to the size of the cover (the exact scaling factor will be irrelevant). Thus $\mathbf{Y}_{0}$ denotes a cover object emitted by the steganographer.

In parts of the paper we will assume a probability distribution for cover and stego objects (even though, as we argue in section 2.1, this distribution is unknowable precisely): the distribution of $\mathbf{Y}_{\beta}$ will be denoted $P_{\beta}$, or if the distribution depends on other parameters $\boldsymbol{\theta}$ then $P_{\beta}^{\boldsymbol{\theta}}$. Thus $P_{0}$ is the distribution of cover objects from the steganographer's source.

\section{STEGANOGRAPHY}

Steganographic embedding in a single grayscale image could be implemented in the real world, with a high degree of undetectability against contemporary steganalysis, if practitioners were to use today's state of art. In this section we begin by outlining that state of art, and highlighting the open problems for its further improvement. However, the same cannot be said of creating a steganographic channel in a stream of multiple objects - which is, after all, the essential aim for systems supporting censorship resistance nor for robust key exchange, and our discussion is mainly of open problems barely treated by the literature.

We begin, in section 2.1, with some results which live purely in the laboratory. They apply to the security model in which the steganographer understands her cover source perfectly, or has exponential amounts of time to wait for a perfect cover. In section 2.2 we move closer to the real world, describing methods which help a steganographer to be less detectable when embedding a given payload. They require, however, the steganographer to know a tractablyoptimizable distortion function, which is really a property of her enemy. Such research was far from the real world until recently, and is moving to practical applicability at the present time. But it does not tell the steganographer whether her size of payload is likely to be detectable; some purely theoretical research is discussed in section 2.3, which gives rules of thumb for how payload should scale as properties of the cover vary, but it remains an open problem to determine an appropriate payload for a given cover. 
In section 2.4 we modify the original steganography model to better account for the repeated nature of communications: if the steganographer wants to create a covert channel, as opposed to a one-shot covert communication, new considerations arise. There are many open research problems in this area. Section 2.5 addresses the key exchange between the steganographer and her participant. The problem is well-understood with a passive warden opponent, but in the presence of an active warden it may even be impossible.

Section 2.6 briefly surveys other ways in which weaknesses may arise in practice, having been omitted from the model, and section 2.7 discusses whether the steganographer can encourage real-world situations favourable to her.

\subsection{The laboratory: perfect steganography}

One can safely say that perfectly secure steganography is now well understood. It requires that the distribution of stego objects be identical to that of cover objects.

In a model where the covers are sequences (usually of fixed length) of symbols from a fixed alphabet, the steganographer fully understands the cover source if they know the distribution of the symbols, including any conditional dependence between them. In such a case, perfect steganography is a coding problem and the capacity or rate (the number of bits per cover symbol) of perfectly secure steganography is bounded by the entropy of the cover distribution. Constructions for such coding have been proposed, including the cases of a distortion-limited sender (the sender is limited in how much the cover can be modified) and even a power-limited active Warden (the Warden can inject a distortion of limited power), for i.i.d. and Markov sources [101].

However, such a model of covers is necessarily artificial. The distinction between artificial and empirical cover sources has been proposed in [14] and is pivotal to the study of steganography in digital media. Artificial sources prescribe a probability distribution from which cover objects are drawn, whereas empirical sources take this distribution as given somewhere outside the steganographic system, which we could call reality. The steganographer can sample an empirical distribution, thereby obtaining projections of parts of reality; she can estimate salient features to devise, calibrate, and test models of reality; but she arguably can never fully know it. The perfect security of the preceding constructions rests on perfect knowledge of the cover source, and any violation of this assumption breaks the security proof. In practical situations, it is difficult to guarantee such an assumption. In other words, secure steganography exists for artificial sources, but we can never be sure if the artificial source exists in practice. More figuratively, artificial channels sit in the corner of the laboratory farthest away from the real world. But they can still be useful as starting points for new theories or as benchmarks.

Perfect steganography is still possible, albeit at higher cost, with empirical cover sources. If (1) secure cryptographic one-way functions exist, (2) the steganalyst is at most equally limited in her knowledge about the cover source as the steganographer, and (3) the cover source can be efficiently sampled, then perfect steganography is possible (the rejection sampler), but embedding requires an exponential number of samples in the message length [14, Ch. 3]. Some authors work around the inconvenient embedding complexity by tightening the third assumption and requiring that sampling is efficient conditional to any possible history of transmitted cover objects [41, 85, 44], which is arguably as strong as solving the original steganography problem.

\subsection{Optimal embedding}

If the steganographer has to use imperfect steganography, which does not preserve exactly the distribution of objects, how should she embed to be less detectable? Designing steganography for empirical cover sources is challenging, but there has been great progress in recent years. The steganographer must find a proxy for detectability, which we call distortion. Then message embedding is formulated as source coding with a fidelity constraint [91] - the sender hides her message while minimizing an embedding distortion [58, 79, 39]. As well as providing a framework for good embedding, this permits one to compute the largest payload embeddable below a given embedding distortion, and thus evaluate the efficiency of a specific implementation (coding method).

There are two challenges here: to design a good distortion function, and to find a method for encoding the message to minimize the distortion. We consider the latter problem first.

Early steganographic methods were severely limited by their ability to minimize distortion tractably. The most popular idea was to embed the payload while minimizing the number of changes caused (matrix embedding [21]). Counting the embedding changes, however, implicitly assumes that each change contributes equally to detectability, which does not coincide with experimental experience.

The idea of adaptive embedding, where each cover element is assigned a different embedding cost, dates to the early days of digital steganography [31]. A breakthrough technique was to use syndrome-trellis codes (STCs) [29], which solve certain versions of the adaptive embedding problem. The designer defines an additive distortion between the cover and stego objects in the form

$$
D(\mathbf{X}, \mathbf{Y})=\sum_{i} \rho_{i}\left(\mathbf{X}, Y_{i}\right)
$$

where $\rho_{i} \geq 0$ is a local distortion measure that is zero if $Y_{i}=X_{i}$, and then embeds her message using STCs, which minimize distortion between cover and stego objects for a given payload.

STCs only directly solve the embedding problem for distortion functions that are additive in the above sense, or where an additive approximation is suitable. Recently, suboptimal coding schemes able to minimize non-additive distortion functions were proposed, thereby modelling interactions among embedding changes, using the Gibbs construction. This can be used to implement embedding with an arbitrary distortion that can be written as a sum of locally supported potentials [27]. Unfortunately, such schemes can only reach the rate-distortion bound for additive distortion measures. Moving to wider classes of distortion function, along with provably optimal and practical coding algorithms, is an area of current research.

Open Problem 1 Design efficient coding schemes for nonadditive distortion functions.

How, then, to define the distortion function? For the steganographer, the distortion function is a property of her enemy, the steganalyst. If she were to know what steganalysis she is up against then it would be tempting to use the same feature representation as her opponent, defining $D(\mathbf{X}, \mathbf{Y})=\|f(\mathbf{X})-f(\mathbf{Y})\|$, where $f$ is the feature extrac- 
tion function. Such a distortion function, however, is nonadditive and non-local in just about all feature spaces used in steganalysis, which typically include histograms and highorder co-occurrences, created by a variety of local filters. One option is to make an additive approximation. Another, proposed in [27], is to create an upper bound to the distortion function, by writing its macroscopic features as a sum of locally-supported functions (for example, the elements of a co-occurrence matrix can be written as the sum of indicator functions operating on pairs of pixels). In such a case, the distortion function can be bounded, using the triangle inequality, leading to a tractable objective function for STCs.

Even if the coding problem can be solved, such embedding presupposes knowledge of the right distortion function. An alternative is to design a distortion function which reflects statistical detectability (against an optimal detector), but this is difficult to do, let alone the constraints of our current coding techniques. First attempts in these directions adjusted parameters of a heuristically-defined distortion function, to give the smallest margin between classes in a selected feature space [28]. However, unless the feature space is a complete statistical descriptor of the empirical source [61], such optimized schemes may, paradoxically, end up being more detectable [65], which brings us back to the main and rather difficult problem: modelling the source.

Open Problem 2 Design a distortion function relating to statistical detectability, e.g. via KL divergence (sect. 2.3).

Design of heuristic distortion functions is currently a highly active research direction. It seems that the key is to assign high costs to changes to areas of a cover which are "predictable" from other parts of the stego object or other information available to the steganalyst. For example, one may use local variance to compute pixel costs in spatial domain images [97]. The embedding algorithm HUGO [79] uses an additive approximation of a weighted norm between cover and stego features in the SPAM feature space [78], with high weights assigned to well-populated feature bins and low weights to sparsely populated bins that correspond to more complex content. An alternative distortion function called WOW (Wavelet Obtained Weights) [40] uses a bank of directional high-pass filters to assign high distortion where the content is predictable in at least one direction. It has been shown to resist steganalysis using rich models [35]. A further development is published in these proceedings.

One can expected that future research will turn to computer vision literature, where image models based on Markov Random Fields [102, 87, 94] are commonly trained and then utilized in various Bayesian inference problems.

In the domain of grayscale JPEG images, by far the most successful paradigm is to minimize the distortion w.r.t. the raw, uncompressed cover image, if available [58, 86, 100, 43]. In fact, this "side-informed embedding" can be applied whenever the sender possesses a higher-quality "precover" that was quantized to obtain the cover. Currently, the most secure embedding method for JPEG images that does not use any side information is the heuristically-built Uniform Embedding Distortion [39] that substantially improved the previous state of the art: the nsF5 algorithm [36].

Open Problem 3 Distortion functions which take account of side information.

We conclude by highlighting the scale of research advances seen in embedding into grayscale (compressed or uncom- pressed) images. The earliest aims to reduce distortion attempted to correct macroscopic properties (e.g., an image histogram) by compensating embedding changes with additional correction changes, but in doing so made themselves more detectable, not less. We have progressed through a painful period where distortion minimization could not tractably be performed, to the most recent adaptive methods. However, we know of no literature addressing the parallel problems:

Open Problem 4 Distortion functions for colour images and video, which take account of correlations in these media.

Network steganography has received substantial attention from the information theory community through the analysis of covert timing channels [6, 98], which uses delays between network packets to embed the payload. However, the implementations are usually naive, using no distortion with respect to delays of normal data $[16,12]$. The design of the embedding schemes focuses mainly on robustness with respect to the network itself, because network steganography is an active steganography problem. To the knowledge of the authors, the only work that considers a statistical distortion between normal and stego traffic is provided in [9].

\subsection{Scaling laws}

In this section we discuss some theory which has relevance to real-world considerations. These results rest on some information theory: the data processing theorem for Kullback-Leibler (KL) divergence [69]. We are interested in KL divergence between cover objects and stego objects, which we will denote $D_{\mathrm{KL}}\left(P_{0} \| P_{\beta}\right)$. Cachin [17] described how an upper bound on this KL divergence implies an upper bound on the performance of any detector; we do not repeat the argument here. What matters is that we can analyze KL divergence, for a range of artificial models of covers and embedding, and obtain interesting conclusions.

As long as the family of distributions $P_{\beta}^{\theta}$ satisfies certain smoothness assumptions, for fixed cover parameters $\boldsymbol{\theta}$ the Taylor expansion to the right of $\beta=0$ is

$$
D_{\mathrm{KL}}\left(P_{0}^{\boldsymbol{\theta}} \| P_{\beta}^{\boldsymbol{\theta}}\right) \sim \frac{n}{2} \beta^{2} I^{\boldsymbol{\theta}}(0),
$$

where $n$ is the size of the objects and $I^{\boldsymbol{\theta}}(0)$ is the so-called Fisher information. This can be interpreted in the following manner: in order to keep the same level of statistical detectability as the cover length $n$ grows, the sender must adjust the embedding rate so that $n \beta^{2}$ remains constant. This means that the total payload, which is $n \beta$, must be proportional to $\sqrt{n}$. This is known as the square root law of imperfect steganography. Its effects were observed experimentally long before it was formally discovered first within the context of batch steganography [50], experimentally confirmed [57], and finally derived for sources with memory [30], where the reader should look for a precise formulation.

The law also tells us that the proper measure of secure payload is the constant of proportionality, $I^{\boldsymbol{\theta}}(0)$, the Fisher information. The larger $I^{\theta}(0)$, the smaller the secure payload that can be embedded and vice versa. When practitioners design their steganographic schemes for empirical covers, one can say that they are trying to minimize $I^{\boldsymbol{\theta}}(0)$, and it would be of immense value if the Fisher information could be determined for practical embedding methods. But it depends heavily on the cover source, and particularly on the likelihood of rare covers, which by definition is difficult 
to estimate empirically, and there has as yet been limited progress in this area, benchmarking [26] and optimizing [53] simple embedding only in restrictive artificial cover models. Open Problem 5 Robust empirical estimate of steganographic Fisher information.

What is remarkable about the square root law is that, although both asymptotic and proved only for artificial sources, it is robust and manifests in real life. This is despite the fact that practitioners detect steganography using empirical classifiers which are unlikely to approach the bound given by KL divergence, and the fact that empirical sources do not match artificial models. Beware, though, that it tells us how the secure payload scales when changing the number of cover elements, without changing their statistical properties - e.g. when cropping homogeneous images or creating a panorama by simple composition - but not when a cover is resized, because resizing changes the statistical properties of the cover pixels by weakening (if downscaling without antialiasing) or strengthening (if using a resampling kernel) their dependencies.

We can still say something about resized images, if we accept a Markov chain cover model. When nearest neighbour resizing is used, one can compute numerically $I^{\theta}(0)$ as a function of the resizing factor (which should be thought of as part of $\boldsymbol{\theta}$ ) [64]. This allows the steganographer to adjust her payload size with rescaling of the cover, and the theory aligns robustly with experimental results.

Open Problem 6 Derivation of Fisher information for other rescaling algorithms, and richer cover models.

Finally, one can ask about the impact of quantization. This is relevant as practically all digital media are obtained by processing and quantizing the output of some analogue sensor, and a JPEG image is obtained from a raw image by quantizing the real-valued output of a transform. For example, how much larger payload can one embed in 10bit grayscale images than in 8-bit? (Provided that both bit depths are equally plausible on the channel.) How much more data can be hidden in a JPEG with quality factor 98 than quality factor 75 ? We can derive (in an appropriate limit) $I^{\theta}(0) \sim \triangle^{s}$, where $\triangle>0$ is the quantization step and $s$ is the quantization scaling exponent that can be calculated from the embedding operation and the smoothness of the unquantized distribution [32]. In general, the smoother the unquantized distribution, the larger $s$ is and the smaller the Fisher information (larger secure payload). The exponent $s$ is also larger for embedding operations that have a smoothing effect. Because the KL divergence is an error exponent, quantization has a profound effect on security. The experiments in [32] indicate that even simple LSB matching may be practically undetectable in 10-12 bit grayscale images. However, unlike the scaling predicted by the square root law, since the result for quantization depends strongly on the distribution of the unquantized image, it cannot quantitatively explain real life experiments.

\subsection{Multiple objects}

Simmons' 1983 paper used the term "subliminal channel", but the steganography we have been describing is not fully a channel: it focused on embedding a certain length payload in one cover object. For a channel, there must be infinitely many stego objects (perhaps mixed with infinitely many innocent cover objects) transmitted by the steganographer.
How do we adapt steganographic methods for embedding in one object to embedding in many? How should one allocate payload between multiple objects? There has been very little research on this important problem, which is particularly relevant to hiding in network channels, where communication is naturally repeated.

In some versions of the model, this is fundamentally no different from the simple steganography problem in one object. Take the case, for example, where the steganographer has a fixed number of covers, and decides how to allocate payload amongst them (the batch steganography problem posed in [48]). Treating the collection as a single large object is possible if the full message and all covers are instantly available and go through the same channel (e.g., stay on the same disk as a steganographic file system). In principle, this reduces the problem to what has been said above. It is worth pointing out that local statistical properties are more likely to change between covers than between symbols within one cover. However, almost all empirical practical cover sources are heterogeneous (non-stationary): samplers and distortion functions have to deal with this fact anyway. And knowing the boundaries between cover objects is just another kind of side information.

The situation is more complicated in the presence of realtime constraints, such as requirements to embed and communicate before the full message is known or before all covers are drawn. This happens, for example, when tunnelling bilateral protocols through steganographic channels. Few publications have addressed the stream steganography problem (in analogy to stream ciphers) [31, 52]. One interesting result is known for payload allocation in infinite streams with imperfect embedding (and applies only to an artificial setup where distortion is exactly square in the amount of payload per object): the higher the rate that payload is sent early, the lower the eventual asymptotic square root rate [52].

A further generalization is to replace the "channel" by a "network" communications model, where the steganographer serves multiple channels, each governed by specific cover source conventions, and with realtime constraints emerging from related communications. Assuming a global passive steganalyst who can relate evidence from all communications, this becomes a very hard instance of a steganography problem, and one that seems relevant for censorshipresistant multiparty communication or to tunnel covert collaboration [10].

Open Problem 7 Theoretical approaches and practical implementations for embedding in multiple objects in the presence of realtime constraints.

\subsection{Key exchange}

A curious problem in a steganographic environment is that of key exchange. If a reliable steganographic system exists, can parties use that channel to communicate, without first sharing a secret key? In the cryptographic world, Alice and Bob use a public-key cryptosystem to effect a secret key exchange, and then communicate with a symmetric cipher; one would assume that some similar exchange would enable communication with a symmetric stegosystem. However, a steganographic channel is fundamentally different from a traditional communications channel, due to its extra constraint of undetectability. This constraint also limits our ability to transmit datagrams for key establishment. 
Key exchange has been addressed with several protocols and, paradoxically, negative results. The first protocol for key exchange under a passive warden [7] was later augmented to survive an active warden [8]. Here Alice and Bob use a public embedding key to transmit traditional key exchange datagrams: first a public encryption key, and then a session key encrypted with that public key. These datagrams are visible to the warden, but they are designed to resemble channel noise so that the warden cannot tell if the channel is in use. This requires a complete lack of observable structure in the keys.

To prevent an active warden from altering the datagrams, the public embedding key is made temporarily private: first a datagram is sent with a secret embedding key, and then this key is publicly broadcast after the stego object passes the warden. In [22] it was argued that a key broadcast is not allowed in a steganographic setting, but that a key could be encoded as semantic content of a cover.

This may seem to settle the problem, but recent results argue that these protocols, and perhaps any such protocols, are practically impossible because the datagrams are sensitive to even a single bit error. If an active warden can inflict a few errors, we have a problem due to a fundamental difference between steganographic and traditional communication channels: we cannot use traditional error correction, because its presence is observable structure that betrays the existence of a message. In [71], it was shown that this fragility cannot be fixed in general: most strings are a few surgical errors away from a failed transmission; this allows key exchange to be derailed with an asymptotically vanishing error rate. It is not clear who will have the upper hand in practice: an ever-vigilant warden can indefinitely postpone key exchange with little error, but a brief opportunity to transmit some uncorrupted datagrams results in successful key transmission, whereupon the warden loses.

A final problem in steganographic key exchange is the state of ignorance of sender and receiver, and the massive computational burden this implies. Because key datagrams must resemble channel noise, nobody can tell if or when they are being transmitted; by the constraints of the problem, neither Alice nor the warden can tell if Bob is participating in a protocol, or innocently transmitting empty covers. This is solved by brute force: Bob assumes that the channel noise of every image is a public key, and sends a reply. Alice makes similar assumptions, both repeatedly attempting to generate a shared key until they produce one that works.

Open Problem 8 Is this monstrous amount of computation necessary, or is there a protocol with more efficient guesswork to allow Alice and Bob to converge on a key?

\subsection{Basic security principles}

Finally, even when a steganographic method is secure, its security can be broken if there is information leakage of the secret key, or of the steganography software. We recall some basic principles that should be followed by the steganographer, in order to avoid security pitfalls.

- Her embedding key must be long enough to avoid exhaustion attacks [34], and any pseudorandom numbers generated from it must be strong.

- Whenever she wants to embed a payload in several images, she must avoid using the same embedding locations for each. Otherwise the steganalyst can use noise residuals to estimate the embedding locations, reducing the entropy of the secret key [51]. One way to force the locations to vary is to add a robust hash of the cover to the seed.

- She must act identically to any casual user of the communication channel, which implies hiding also the use of steganographic software, and deleting temporary cover and stego objects. An actor that performs cover selection by emitting only contents that are known to be difficult to analyze (such as textured images) can seem suspicious in itself.

Open Problem 9 How to perform cover selection, if at all? How to detect cover selection?

- She has to beware of the pre- and post-processing operations that can be associated with embedding. Double compression can be easily detected [80] and forensic details, such as the ordering of different parts of a JPEG file, can expose the processing path [38].

- She should benchmark her embedding appropriately. In the case of digital images for example, it is not because the software produces imperceptible embedding that the payload is undetectable. Image quality metrics such as the PSNR and psychovisual metrics are of little interest in steganography. - Her device capturing the cover should be trusted, and contents generated from this device should also stay hidden. Covers must not be re-used.

Several general principles should be kept in mind when designing a secure system. These include:

- The Kerckhoffs Principle, that a system should remain secure under the assumption that the adversary knows the system, although interpretations for steganography differ in whether this includes knowledge of the cover source or not. - The Usability Principle (also due to Kerckhoffs), that a system should be easy for a layperson to use correctly. For example, steganographic software should enforce a square root law rather than expecting an end user to apply it.

- The Law of Leaky Abstractions [93], which requires us to be aware of, for example, statistical models of cover sources, assumptions about the adversary, or the abstraction of steganography as a generic communication channel. Even if we have provable security within the model, reality may deviate from the model in a way that causes a security weakness.

- The fact that steganographic channels are not communications channels in the traditional sense, and their limitations are different. Challenges of capacity, fidelity, and key exchange must be examined anew.

Open Problem 10 Are there abstractions that hold for steganography? Are its building blocks securely composable?

\subsection{Engineering the real world for steganography}

If we perfectly understood our cover sources, secure steganography would reduce to a coding problem. Engineering secure steganography for the real world is so difficult precisely because it requires us to understand the real world as well as our artificial models. If there is a consensus that the real world needs secure steganography, a completely different approach could be to engineer the real world so that parts of it match the assumptions needed for security proofs. This implies changing the conventions, via protocols and norms, towards more randomness in everyday communications, so that more artificial channels knowingly exist in the real world. For example, random nonces in certain protocols, or synthetic pseudorandom textures in video-games (if 
implemented with trustworthy randomness) already provide opportunities for steganographic channels. Adding more of these increases the secure capacity ([23] proposes a concrete system). But this approach creates new challenges, many outside the domain of typical engineering, such as the social coordination problem of giving up bandwidth across the board to protect others' communication relations, or the difficulty of verifying the quality of randomness.

Open Problem 11 Technical and societal aspects of inducing randomness in communications to simplify steganography.

\section{STEGANALYSIS}

Approaches to the steganalysis problem depend heavily on the security model, and particularly on the steganalyst's knowledge about the cover source and the behaviour of his opponent. The most studied models are quite far from real-world application, and (unlike steganography) most researchers would agree that state of the art steganalysis could not yet be used effectively in the real world.

Laboratory conditions apply in section 3.1, where we assume that the steganalyst has perfect knowledge of (1) the cover source, (2) the embedding algorithm used by the steganographer, and (3) which object they should examine. This is as unrealistic as the parallel conditions in section 2.1 , but the laboratory work provides a conservative attack model, and still gives interesting insights into practice. Almost all current steganalysis literature adheres to the model described in section 3.2, which weakens (1) so that the steganalyst can only learn about the cover source by empirical samples; it is usually assumed that something similar to (2) still holds, and (3) must hold. This line of steganalysis research, which rests on binary classification, is highly refined, but weakening even slightly the security model leads to difficult problems about learning.

In section 3.3 we ask how a steganalyst could widen the application of binary classifiers by using them in combination, and in 3.4 by moving to a model with complete ignorance of the embedding method (and empirical knowledge of the covers). Although these problems are known in machine learning literature, there have been few steganalysis applications.

In section 3.5 we open the model still further, weakening assumption (3), above, so that the steganalyst no longer knows exactly where to look: first, against one steganographer making many communications, and then when monitoring an entire network. This parallels section 2.4, and reveals an essentially game-theoretic nature of steganography and steganalysis, which is the topic of section 3.6. Again, there are many open problems.

Finally, section 3.7 goes beyond steganalysis, to ask what further information can be gleaned from stego objects.

\subsection{Optimal detection}

The most favourable scenario for the steganalyst occurs when the exact embedding algorithm is known, and there is a statistical model for covers. In this case it is possible to create optimal detection using statistical decision theory, although the framework is not (yet) very robust under less favourable conditions.

The inspected medium $\mathbf{Y}=\left(Y_{1}, \ldots, Y_{N}\right)$ is considered as a set of $N$ digital samples (not necessarily independent), and $P_{\beta}^{\theta}$ the distribution of stego object $\mathbf{Y}_{\beta}$, after embedding at rate $\beta$. We are separating one parameter controlling the embedding, $\beta$, from other parameters of the cover source $\boldsymbol{\theta}$ which in images might include size, camera settings, colour space, and so on.

When the embedding rate $\beta$ and all cover parameters $\boldsymbol{\theta}$ are known, the steganalysis problem is to choose between the following hypotheses: $\mathcal{H}_{0}=\left\{\mathbf{Y} \sim P_{0}^{\boldsymbol{\theta}}\right\}$ vs $\mathcal{H}_{1}=\left\{\mathbf{Y} \sim P_{\beta}^{\boldsymbol{\theta}}\right\}$. These are two simple hypotheses, for which the NeymanPearson Lemma [70, Th. 3.2.1] provides a simple way to design an optimal test, the Likelihood Ratio Test (LRT):

$$
\delta^{\mathrm{LRT}}=\left\{\begin{array}{l}
\mathcal{H}_{0} \text { if } \Lambda(\mathbf{Y})=\frac{P_{\beta}^{\theta}[\mathbf{Y}]}{P_{0}^{\theta}[\mathbf{Y}]}<\tau \\
\mathcal{H}_{1} \text { if } \Lambda(\mathbf{Y})=\frac{P_{\beta}^{\theta}[\mathbf{Y}]}{P_{0}^{\theta}[\mathbf{Y}]} \geq \tau
\end{array}\right.
$$

with $\Lambda$ the likelihood Ratio (LR) and $\tau$ a decision threshold.

The LRT is optimal in the following sense: among all the tests which guarantee a maximum false-alarm probability $\alpha \in(0,1)$ the LRT maximizes the correct detection probability. This is not the only possible measure of optimality, which we return to in section 3.6.

Accepting, for a moment, the optimal detection framework, we can deduce some interesting "laboratory" results. Assume that pixels from a digital image are i. i. d.: then the statistical distribution $P^{\theta}$ of an image is its histogram. If cover samples follow a Gaussian distribution $X_{i} \sim \mathcal{N}\left(\mu_{i}, \sigma_{i}^{2}\right)$, it has been shown [107] that the LR for the LSB replacement scheme can be written: $\Lambda(\mathbf{Y}) \propto \sum_{i}\left(y_{i}-\bar{y}_{i}\right)\left(y_{i}-\mu_{i}\right) / \sigma_{i}^{2}$, where $\bar{k}=k+(-1)^{k}$ is the integer $k$ with flipped LSB. This LR is similar to the well-known Weighted Stego-image statistic $[33,54]$ and justifies it post hoc as an optimal hypothesis test. Similarly, the LR for the LSB matching scheme can be written $[18]: \Lambda(\mathbf{Y}) \propto \sum_{i}\left(\left(y_{i}-\mu_{i}\right)^{2}-\frac{1}{12}\right) / \sigma_{i}^{4}$. This shows that optimal detection of LSB matching is essentially based on pixel variance. Particularly since LSB matching has the effect of masking the true cover variance, this explains it has proved a tougher nut to crack than LSB replacement.

However, the assumption that pixels can be modelled as i. i. d. random variables is unrealistic. Similarly, the model of statistically independent pixels following a Gaussian distribution (with different expectation and variance) is of limited interest in the real world.

The description of the steganalysis problem in the framework of hypothesis testing theory emphasizes the practical difficulties. First, it seems highly unlikely that the embedding rate $\beta$ would be known to a steganalyst, unless they already know that steganography is being used. And when $\beta$ is unknown the design of an optimal statistical test becomes much harder because the alternative hypothesis $\mathcal{H}_{1}$ is composite: it gathers different hypotheses, for each of which a different most powerful test exists.

There are two approaches to overcome this difficulty: design a test which is locally optimal around a target embedding rate [19, 107] (again these tests rely on a statistical model of pixels); or design a test which is universally optimal for any embedding rate [18] (unfortunately their optimality assumptions are seldom met outside "the laboratory").

Open Problem 12 Theoretically well-founded, and practically applicable, detection of payload of unknown length.

Second, it is also unrealistic to assume that the vector parameter $\boldsymbol{\theta}$, which defines the statistical distribution of the whole inspected medium, is perfectly known. In practice, 
these parameters are unknown and would have to be estimated using a model. Here one could employ the Generalized Likelihood Ratio Test (GLRT), which estimates unknown parameters in the LRT by the method of maximum likelihood. Unfortunately, maximum likelihood estimators again depend on a particular models of covers, and furthermore the GLRT is not usually optimal.

Although models of digital media are not entirely convincing, a few have been used for steganalysis, e.g. [20], as well as models of camera post-acquisition processing such as demosaicking and colour correction [95]. Much is unexplored.

Open Problem 13 Apply models from the digital imaging community, which do not require independence of pixels, to the optimal detection framework.

However, it is sobering to observe that a well-developed detector based on testing theory and Laplacian model of DCT coefficients [106] performs poorly in practice compared to the rather simple WS detector adapted to the JPEG domain [13]. As we have repeatedly stated, digital media steganography is a particularly difficult domain in which to understand the covers.

\subsection{Binary classification}

Absent a model of covers, currently the best image steganalyzers are built using feature-based steganalysis and machine learning. They rest on the assumption that the steganalyst has some samples from the steganographer's cover source, so that its statistical properties can be learned, and also that they can create or otherwise obtain stego objects from these covers (for example by knowing the exact embedding algorithm). Typically, one starts by representing the media using a feature of a much smaller dimensionality, usually designed by hand using heuristic arguments. Then, a training database is created from the cover and stego examples, and a binary classifier is trained to distinguish the two classes.

Machine-learning steganalysis is fundamentally different from statistical signal processing approaches because one does not need to estimate the distribution of cover and stego images. Instead, this problem is replaced with a much simpler one: merely to distinguish the two classes. Thus, one can build classifiers that use high-dimensional features even with a limited number of training examples. When trained on the correct cover source, feature-based steganalysis usually achieves significantly better detection accuracy than analytically derived detectors (with the exception of LSB replacement).

There are two components to this approach: the features, and the classification algorithm.

Image steganalysis features have been well-studied in the literature. In the spatial domain, one usually starts by computing noise residuals, by creating and then subtracting an estimate of each cover pixel using its neighbours. The pixel predictors are usually built from linear filters, such as local polynomial models or 2-dimensional neighbourhoods, and can incorporate nonlinearity using the operations of maximum and minimum. The residuals improve the SNR (stego signal to image content). Typically, residuals are truncated and quantized into $2 T+1$ bins, and the final feature vector is the joint probability mass function (co-occurrence) or conditional probability distribution (transition matrix) of $D$ neighbouring quantized residuals [78]. The dimensionality of this feature vector is $(2 T+1)^{D}$, which quickly grows especially with the co-occurrence order $D$, though it can somewhat be reduced by exploiting symmetry.

In the JPEG domain, one can think of the DCT coefficients already as residuals and form co-occurrences directly from their quantized values. Since there exist dependencies among neighboring DCT coefficients both within a single $8 \times 8$ block as well as across blocks, one usually builds features as two-dimensional intra-block and inter-block cooccurrences [60]. It is also possible to build the co-occurrences only for specific pairs of DCT modes [62]. A comprehensive list of source code for feature vectors for raw and compressed images, along with references, is available at [2]. The current state of art in feature sets are unions of co-occurrences of different filter residuals, so-called rich models. They tend to be high-dimensional (e.g., 30000 or more) but they also tend to exhibit the highest detection accuracy [35, 63].

We note that, in parallel to the steganography situation, steganalysis literature is mostly specialized to grayscale images: there exists only a little literature on steganalysis in video, e.g. [15, 47], and for various kinds of network traffic analysis [16, 104, 12]. The latter methods only use basic statistics such as the variance of inter-packet delays or quantiles of differences between arrival times. There is scope to transfer lessons from grayscale image steganalysis to these domains.

Open Problem 14 Design features for colour images and video, which take account of correlations in these media, and rich features for network steganalysis.

Another problem specific to steganalysis of network traffic is the difficulty of acquiring large and diverse data sets.

The second component, the machine learning tool, is a very important part. When the training sets and feature spaces are small, the tool of choice is the support vector machine (SVM) [88] with Gaussian kernel, and this was predominant in the literature to 2011. But with growing feature dimensionality, one also needs larger training sets, and it becomes computationally unfeasible to search for hyperparameters. Thus, recently, simpler classifiers have become more popular. An example is the ensemble classifier [66], a collection of weak linear base learners trained on random subspaces of the feature space and on bootstrap samples of the training set. The ensemble reaches its decision by combining the decisions of individual base learners. (In contrast, decision trees are not suitable for steganalysis, because among the features there is none that is strong alone.) When trying to move the tools from the laboratory to the real world, one likely needs to further expand the training set, which may necessitate online learning such as the simple perceptron and its variants [72]. There has been little research in this direction. Online learning also requires fast extraction of features, which is in tension with the trend towards using many different convolution filters.

Although highly refined, the paradigm of training a binary classifier has some limitations. First, it is essentially a binary problem, which presupposes that the steganalyst knows exactly the embedding method and payload size used by their attacker. Dealing with unknown payload sizes has been approached in two ways: quantitative steganalysis (see section 3.7), or effectively using a uniform prior by creating the stego training set with random payload lengths [77]. An unknown embedding method is more difficult and changes to the problem to either a multi-class classification (com- 
putationally expensive [76]) or one-class anomaly detection (section 3.4).

A more serious weakness is that the classifier is only as good as its training data. Although it is possible, in the real world, that the steganalyst has access to the steganographer's cover source (e.g. he arrests her and seizes her camera), it seems an unlikely situation. Thus the steganographer must train the classifier on some other source. This leads to cover source mismatch, and the resulting classifier suffers from decreased accuracy. The extent of this decrease depends on the features and the classifier, in a way not yet fully understood. It is fallacious to try to train on a large heterogeneous data set as somehow "representative" of mixed sources, because it guarantees a mismatch and may still be an unrepresentative mixture.

Machine learning literature refers to the problem of domain adaptation, which could perhaps be applied to this challenge.

$\overline{\text { Open Problem } 15 \text { Attenuate the problems of cover source }}$ mismatch.

A final issue in moving machine-learning steganalysis to the real world is the measure of detection accuracy. Popular measures such as $\min \frac{1}{2}\left(P_{F P}+P_{F N}\right)$ correspond to the minimal Bayes risk under equally likely cover and stego images, which is doubtful in practice. Indeed, one might expect that real-world steganography is relatively rarely observed, so real-world steganalysis should be required to have very low false positive rates, yet steganalysis with very low false positive rates has hardly been studied. Even having a reliable false positive rate would be a good start, and there has been some research designing detectors with constant false-alarm rate (CFAR) [68], but it relies on artificial cover models and is also vulnerable to cover source mismatch. It should be noted that establishing classification error probabilities remains unsolved in general [90].

\subsection{Adaptive classification}

Suppose that, for different cover parameters $\boldsymbol{\theta}$, we have trained different specialized binary classifiers. One possibility is to select the optimal classifier for each observed stego object. This approach has been used to tackle images which have double JPEG compression, and those with different JPEG quality factors (in the absence of quantizationblind features, such images have to be considered as coming from completely different sources) [76]. A similar approach specializing detectors to different covers has been pursued in [42].

This is a special case of fusion, where multiple classifiers have their answers combined in some weighted fashion. It presupposes that the cover parameters $\boldsymbol{\theta}$ can reliably be estimated from the observed stego image, and that training data was available for all reasonable combinations of parameters. It is also very expensive in terms of training. In machine learning this architecture is known as a mixture of experts [105].

$\overline{\text { Open Problem } 16 \text { Apply other fusion techniques to ste- }}$ ganalysis.

\subsection{Universal steganalysis}

It is not always realistic to assume that the embedder knows anything about the embedding algorithm used by the steganographer. Universal steganalysis focuses on such a scenario, assuming that the steganalyst can draw empirically from the cover source but is otherwise ignorant. Despite being almost neglected by the community, such a problem is important for deployment of steganalysis in the real world.

Universal steganalysis considers the following hypothesis test: $\mathcal{H}_{0}=\left\{\mathbf{Y} \sim P_{0}^{\boldsymbol{\theta}}\right\}$ vs $\mathcal{H}_{1}=\left\{\mathbf{Y} \nsim P_{0}^{\boldsymbol{\theta}}\right\}$. We can distinguish two cases: either the cover source is entirely known to the detector $\left(\boldsymbol{\theta}\right.$ is known and $\mathcal{H}_{0}$ is simple), or not (both hypotheses are composite). The first version of the problem is unrealistic in the real world, for the reasons we previously cited. The second shows that detector design is about modelling a cover source, and practical approaches resort to modelling the distribution of cover images in a space determined by steganographic features. In comparison with the binary hypothesis testing scenario of section 3.2, this problem is much more difficult, because learning a probability distribution is unavoidably more difficult than learning a classifier [96]. We must expect that universal steganalyzers have inferior performance to targeted binary classifiers. In fact it is not straightforward to benchmark universal steganalysis, because there is no well-defined alternative hypothesis class from which to test for false negatives.

Universal steganalysis can be divided into two types: supervised and unsupervised. The former uses samples from the cover-source to create the cover model, e.g. by using one-class support vector machines [88] designed to solve the above hypothesis test under a false positive constraint. This approach has been investigated in [82, 73]. Obviously, the accuracy of supervised steganalysis is limited if the training data is not perfectly representative of the steganographer's cover source and, if mismatched, the accuracy might be as bad as random guessing.

Unsupervised universal steganalysis tries to circumvent the problem of model mismatch by postponing building a cover model until the classification phase. It analyses multiple images at once, assuming that most of them are covers, and is therefore a form of outlier detection. To our knowledge there is no literature dealing with this scenario in steganalysis, though there are works dealing with it on the level of actors, treated in section 3.5.

Open Problem 17 Unsupervised universal steganalysis.

The accuracy of universal steganalysis is to a large extent determined by the steganographic features, and features suitable for binary classification are not necessarily right for universal steganalysis. The features should be sensitive to changes caused by embedding, yet insensitive to variations between covers (including perhaps unnatural but non-steganographic processing techniques). Particularly in the case of unsupervised learning, the latter condition requires them to have low dimension, because unsupervised learning cannot learn to ignore irrelevant noise. A small number of features also facilitates training of supervised detectors, as it decreases the required number of samples to learn the probability distribution. An unstudied problem is therefore:

Open Problem 18 Design of features suitable for universal steganalysis.

\subsection{Pooled and multi-actor steganalysis}

So far, the security models have assumed that the steganalyst has one object to classify, or if they have many then they know exactly which one to look at. This is highly un- 
realistic and if steganalysis is to move to the real world it will have to address the problem of pooled steganalysis [48]: combining evidence from multiple objects to say whether they collectively contain payload. It is in opposition to the steganographic channel of section 2.4.

Although posed in 2006, there has been little success in attacking this problem. One might say that it is no different to binary steganalysis: simply train a classifier on multiple images. But there are many practical problems to overcome: should the feature set be the sum total of features from individual images (if so, this loses information), or concatenated (in which case how does one impose symmetry under permutation)? To our knowledge, there has been no such detector proposed in the literature, except for simple examples studied when the problem was first posed [48, 49].

A related problem which, to the best of our knowledge, has never been studied is sequential detection. When inspecting VOIP traffic, for instance, it would be interesting to perform online detection. The theoretically optimal detection is more complex because time-to-decision also has to be taken into account. The statistical framework of sequential hypothesis tests should be applicable [99].

Open Problem 19 Any detector for multiple objects, or based on sequential hypothesis tests.

We can widen the steganalysis model still further, to a realistic scenario relevant to network monitoring, if the steganalyst does not know even which user to examine. In this situation the steganalyst intercepts many objects each from many actors (e.g. social network users); their problem is to determine which actor(s), if any, are using steganography in some or all of their images.

This is the most challenging version of steganalysis, but recent work $[56,55]$ has shown that the size of the problem can be turned to the steganalyst's advantage: by calibrating the behaviour of actors (as measured through steganalysis features) by the behaviour of the majority, steganographers can potentially be determined in an unsupervised and universal way. It amounts to an anomaly detection where the unit is the actor, not the individual object. This can be related to unsupervised intrusion detection systems [24].

This is a new direction in steganalysis and we say no more about it here, but highlight the danger of false accusations: Open Problem 20 Can steganographers be distinguished from unusual (non-stego) cover sources, by a detector which remains universal?

\subsection{Game theoretic approaches}

The pooled steganalysis problem exposes an essentially game-theoretic situation. When a (batch) steganographer hides all their payload in one object, a certain type of detector is optimal; when they spread their payload in many objects, a different detector is optimal. These statements can be proved in artificial models and observed in practice. Indeed, the same can be said of single images: if the embedder always hides in noisy areas, the detector can focus their attention there, and vice versa. A parallel situation most likely exists in non-media covers.

Game theory offers an interesting perspective from which to study steganography. If both steganographer and steganalyst know the cover source and are computationally unconstrained, the steganographer can embed perfectly; with a shorter key if the steganalyst is computationally bounded.
If the steganographer is computationally bounded, but not the steganalyst, the best she can do is to minimize the $\mathrm{KL}$ divergence, subject to her constraints. Another way to frame this is that she plays a minimax strategy against the bestpossible detector [45].

This may not add a lot of insight in the lab. But once we step out into the real world, where knowledge of the cover source is incomplete and computational constraints defy finding globally optimal distortion functions or detectors, then game theory becomes very useful. It offers a wealth of solution concepts for situations where no maximin or minimax strategies exist. A popular one is the notion of a Nash equilibrium. It essentially says that among two sets of strategies, one for the steganographer (choice of embedding operation, distortion function, parameters etc.) and one for the steganalyst (feature space, detector, parameters such as local weights, etc.), there exist combinations where no player can improve his or her outcome unilaterally. Although exploitation of game theory for steganography has just begun, and we are aware of only four independent approaches $[25$, $49,75,89]$, it seems to be a promising framework which allows us to justify certain design choices, such as payload distribution in batch steganography or distortion functions in adaptive steganography. This is a welcome step to replace heuristics with (some) rigor in the messy scenarios of limited knowledge and computational power, as we find them in the real world.

However, game theory for steganography is in its infancy, and there are substantial obstacles to be overcome, such as:

Open Problem 21 Find equilibria for practical covers, and transfer insights of game-theoretic solutions from current toy models to the real world.

\subsection{Forensic steganalysis}

Finally, what does the steganalyst do after detecting hidden data in an object? The next steps might be called forensic steganalysis, and only a few aspects have been studied in the literature.

If the aim of the steganalyst is to find targets for further surveillance, or to confirm the existence of already-suspected covert communication, circumstantial evidence such as statistical steganalysis is probably sufficient in itself. But for law enforcement it is probably necessary to demonstrate the content of a message by extracting it, in which case the first step is to determine the embedding algorithm. This problem, largely neglected, has been studied in [81] for JPEG images. The detection of different algorithms based on statistical properties will not be perfect, as methods with similar distortion functions and embedding changes are likely to be confused, but this has not been studied for recent adaptive embedding methods.

Open Problem 22 Can statistical steganalysis recognize different adaptive embedding algorithms?

Some identify a specific implementation by a signature, effectively relying on implementation mistakes $[11,103]$, but this is unsatisfactory in general.

Once the embedding method is known, the next step is a brute-force search for the embedding key. Very little research has been done in this area, though two complementary approaches have been identified: using headers to verify the correctness of a key [84], and comparing statistics along 
potential embedding paths [34] in which the correct key deviates from the rest.

Open Problem 23 Is there a statistical approach to key brute-forcing, for adaptive steganography?

Additionally, forensic steganalysis includes estimation of the length of the hidden message (quantitative steganalysis). This knowledge is useful to prevent "plausible deniability", where the steganographer hides two messages, one of which is not incriminating and can be disclosed if forced. Such a scheme is uncovered if the total embedded payload can be estimated. Quantitative steganalysis is a regression problem parallel to binary classification, and the state of the art applies regression techniques to existing steganalysis features $[83,59]$.

\section{CONCLUSIONS}

Over the last ten years, ad-hoc solutions to steganography and steganalysis problems have evolved into more refined techniques. There has been a disparity in the rate of progress: grayscale images have received most of the attention, which should be transferred to colour images, video, other digital media, and non-media covers such as network traffic. Such transfer would bring both steganography and steganalysis closer to real-world implementation.

For steganography, we have stressed the distortion-minimization paradigm, which only became practical with recent developments in coding. There is no good reason not to use such a technique: there are efficiencies from the coding, and if there is a fear that current distortion functions might make detection paradoxically easier, one can use this feedback to redesign the distortion function, and continue the cycle of development. We expect further advances in coding to widen the applicability of such techniques.

For steganalysis, the binary classification case is well-developed, but there is a need to develop techniques that work with unknown algorithms, multiple objects, and multiple actors. Even the theoretical framework which we have highlighted, that of KL divergence as a fundamental measure of security, has yet to be adapted to these domains.

\section{Acknowledgments}

The work of A. Ker and T. Pevný is supported by European Office of Aerospace Research and Development under the research grant numbers FA8655-11-3035 and FA8655-13-13020, respectively. The work of S. Craver and J. Fridrich is supported by Air Force Office of Scientific Research under the research grant numbers FA9950-12-1-0124 and FA955009-1-0666, respectively. The U.S. Government is authorized to reproduce and distribute reprints for Governmental purposes notwithstanding any copyright notation thereon. The views and conclusions contained herein are those of the authors and should not be interpreted as necessarily representing the official policies, either expressed or implied, of EOARD, AFOSR, or the U.S. Government.

The work of R. Cogranne is funded by Troyes University of Technology (UTT) strategic program COLUMBO. The work of T. Pevný is also supported by the Grant Agency of Czech Republic under the project P103/12/P514.

\section{REFERENCES}

[1] Documents reveal Al Qaeda's plans for seizing cruise ships, carnage in europe. CNN, April 2012. http://edition.cnn.com/2012/04/30/world/ al-qaeda-documents-future/index.html, accessed February 2012.

[2] Feature extractors for steganalysis. http://dde. binghamton.edu/download/feature_extractors/, accessed February 2012.

[3] MIT Technology Review: Steganography. http: //www.technologyreview.com/search/site/ steganography/, accessed February 2012.

[4] Russian spies' use of steganography is just the beginning. MIT Technology Review, July 2010. http://www.technologyreview. com/view/419833/ russian-spies-use-of-steganography-is-just-thebeginning/, accessed February 2012.

[5] D. Alperovitch. Revealed: Operation Shady RAT. McAfee White Paper, 2011. http://www.mcafee.com/us/resources/ white-papers/wp-operation-shady-rat.pdf, accessed February 2012.

[6] V. Anantharam and S. Verdu. Bits through queues. IEEE Trans. Inf. Theory, 42(1):4-18, 1996.

[7] R. Anderson. Stretching the limits of steganography. In Information Hiding, 1st International Workshop, volume 1174 of $L N C S$, pages 39-48. Springer-Verlag, 1996.

[8] R. J. Anderson and F. A. P. Petitcolas. On the limits of steganography. IEEE J. Sel. Areas Commun., 16(4):474-481, 1998.

[9] A. Aviv, G. Shah, and M. Blaze. Steganographic timing channels. Technical report, University of Pennsylvania, 2011.

[10] A. Baliga and J. Kilian. On covert collaboration. In Proceedings of the 9th ACM Multimedia 8 Security Workshop, pages 25-34, 2007.

[11] G. Bell and Y.-K. Lee. A method for automatic identification of signatures of steganography software. IEEE Trans. Inf. Forensics Security, 5(2):354-358, 2010.

[12] V. Berk, A. Giana, G. Cybenko, and N. Hanover. Detection of covert channel encoding in network packet delays, 2005.

[13] R. Böhme. Weighted stego-image steganalysis for JPEG covers. In Information Hiding, 10th International Workshop, volume 5284 of LNCS, pages 178-194. Springer-Verlag, 2007.

[14] R. Böhme. Advanced Statistical Steganalysis. Springer-Verlag, 2010.

[15] U. Budhia, D. Kundur, and T. Zourntos. Digital video steganalysis exploiting statistical visibility in the temporal domain. IEEE Trans. Inf. Forensics Security, 1(4):502-516, 2006.

[16] S. Cabuk, C. E. Brodley, and C. Shields. Ip covert timing channels: design and detection. In Proceedings of the 11th ACM conference on Computer and communications security, pages 178-187. ACM, 2004.

[17] C. Cachin. An information-theoretic model for steganography. In Information Hiding, 2nd International Workshop, volume 1525 of LNCS, pages 306-318. Springer-Verlag, 1998.

[18] R. Cogranne and F. Retraint. An asymptotically uniformly most powerful test for LSB matching 
detection. IEEE Trans. Inf. Forensics Security, 8(3):464-476, 2013.

[19] R. Cogranne, C. Zitzmann, L. Fillatre, F. Retraint, I. Nikiforov, and P. Cornu. Statistical decision by using quantized observations. In International Symposium on Information Theory, pages 1135-1139. IEEE, 2011.

[20] R. Cogranne, C. Zitzmann, F. Retraint, I. Nikiforov, P. Cornu, and L. Fillatre. A locally adapted model of natural images for almost optimal hidden data detection. IEEE Trans. Image Process., 2013. (to appear).

[21] R. Crandall. Some notes on steganography. Steganography Mailing List, 1998. available from http://os.inf.tu-dresden.de/ westfeld/ crandall.pdf.

[22] S. Craver. On public-key steganography in the presence of an active warden. In Information Hiding, 2nd International Workshop, volume 1525, pages 355-368, 1998.

[23] S. Craver, E. Li, J. Yu, and I. Atalki. A supraliminal channel in a videoconferencing application. In Information Hiding, 10th International Workshop, volume 5284 of $L N C S$, pages 283-293.

Springer-Verlag, 2008.

[24] D. E. Denning. An intrusion-detection model. IEEE Trans. Softw. Eng., SE-13(2):222-232, 1987.

[25] M. Ettinger. Steganalysis and game equilibria. In Information Hiding, 2nd International Workshop, volume 1525 of $L N C S$, pages 319-328. Springer-Verlag, 1998.

[26] T. Filler and J. Fridrich. Fisher information determines capacity of $\epsilon$-secure steganography. In Information Hiding, 11th International Conference, volume 5806 of $L N C S$, pages 31-47. Springer-Verlag, 2009.

[27] T. Filler and J. Fridrich. Gibbs construction in steganography. IEEE Trans. Inf. Forensics Security, 5(4):705-720, 2010.

[28] T. Filler and J. Fridrich. Design of adaptive steganographic schemes for digital images. In Media Watermarking, Security and Forensics XIII, volume 7880 of Proc. SPIE, pages OF 1-14, 2011.

[29] T. Filler, J. Judas, and J. Fridrich. Minimizing additive distortion in steganography using syndrome-trellis codes. IEEE Trans. Inf. Forensics Security, 6(3):920-935, 2011.

[30] T. Filler, A. D. Ker, and J. Fridrich. The Square Root Law of steganographic capacity for Markov covers. In Security and Forensics of Multimedia XI, volume 7254 of Proc. SPIE, pages 08 1-11, 2009.

[31] E. Franz, A. Jerichow, S. Möller, A. Pfitzmann, and I. Stierand. Computer based steganography: How it works and why therefore any restrictions on cryptography are nonsense, at best. In Information Hiding, 1st International Workshop, volume 1174 of LNCS, pages 7-21. Springer-Verlag, 1996.

[32] J. Fridrich. Effect of cover quantization on steganographic fisher information. IEEE Trans. Inf. Forensics Security, 8(2):361-372, 2013.

[33] J. Fridrich and M. Goljan. On estimation of secret message length in LSB steganography in spatial domain. In Security, Steganography, and Watermarking of Multimedia Contents VI, volume 5306 of Proc. SPIE, pages 23-34, 2004.

[34] J. Fridrich, M. Goljan, and D. Soukal. Searching for the stego key. In Security, Steganography, and Watermarking of Multimedia Contents VI, volume 5306, pages 70-82, 2004.

[35] J. Fridrich and J. Kodovský. Rich models for steganalysis of digital images. IEEE Trans. Inf. Forensics Security, 7(3):868-882, 2011.

[36] J. Fridrich, T. Pevný, and J. Kodovský. Statistically undetectable JPEG steganography: Dead ends, challenges, and opportunities. In Proceedings of the 9th ACM Multimedia \& Security Workshop, pages 3-14, 2007.

[37] J. Giffin, R. Greenstadt, P. Litwack, and R. Tibbetts. Covert messaging through TCP timestamps. In Privacy Enhancing Technologies, volume 2482 of LNCS, pages 194-208. Springer-Verlag, 2002.

[38] T. Gloe. Forensic analysis of ordered data structures on the example of JPEG files. In Information Forensics and Security, 4th International Workshop, pages 139-144. IEEE, 2012.

[39] L. Guo, J. Ni, and Y.-Q. Shi. An efficient JPEG steganographic scheme using uniform embedding. In Information Forensics and Security, 4th International Workshop, pages 169-174. IEEE, 2012.

[40] V. Holub and J. Fridrich. Designing steganographic distortion using directional filters. In Information Forensics and Security, 4th International Workshop, pages 234-239. IEEE, 2012.

[41] N. J. Hopper, J. Langford, and L. von Ahn. Provably secure steganography. In Advances in Cryptology, CRYPTO '02, volume 2442 of $L N C S$, pages 77-92. Springer-Verlag, 2002.

[42] X. Hou, T. Zhang, G. Xiong, and B. Wan. Forensics aided steganalysis of heterogeneous bitmap images with different compression history. In Multimedia Information Networking and Security, 4th International Conference, pages 874-877, 2012.

[43] F. Huang, J. Huang, and Y.-Q. Shi. New channel selection rule for JPEG steganography. IEEE Trans. Inf. Forensics Security, 7(4):1181-1191, 2012.

[44] C. Hundt, M. Liskiewicz, and U. Wölfel. Provably secure steganography and the complexity of sampling. In Algorithms and Computation, volume 4317 of LNCS, pages 754-763. Springer-Verlag, 2006.

[45] B. Johnson, P. Schöttle, and R. Böhme. Where to hide the bits? In J. Grossklags and J. Walrand, editors, Decision and Game Theory for Security, volume 7638 of $L N C S$, pages 1-17. Springer-Verlag, 2012.

[46] D. Kahn. The Codebreakers: The Comprehensive History of Secret Communication from Ancient Times to the Internet. Scribner, revised edition, 1996.

[47] K. Kancherla and S. Mukkamala. Video steganalysis using motion estimation. In International Joint Conference on Neural Networks, pages 1510-1515. IEEE, 2009.

[48] A. D. Ker. Batch steganography and pooled steganalysis. In Information Hiding, 8th 
International Workshop, volume 4437 of LNCS, pages 265-281. Springer-Verlag, 2006.

[49] A. D. Ker. Batch steganography and the threshold game. In Security, Steganography, and Watermarking of of Multimedia Contents IX, volume 6505 of Proc. SPIE, pages 04 1-13, 2007.

[50] A. D. Ker. A capacity result for batch steganography. IEEE Signal Process. Lett., 14(8):525-528, 2007.

[51] A. D. Ker. Locating steganographic payload via ws residuals. In Proceedings of the 10th ACM Multimedia ES Security Workshop, pages 27-32. ACM, 2008.

[52] A. D. Ker. Steganographic strategies for a square distortion function. In Security, Forensics, Steganography, and Watermarking of Multimedia Contents X, volume 6819 of Proc. SPIE, pages 04 1-13, 2008.

[53] A. D. Ker. Estimating the information theoretic optimal stego noise. In Digital Watermarking, 8th International Workshop, volume 5703 of LNCS, pages 184-198. Springer-Verlag, 2009.

[54] A. D. Ker and R. Böhme. Revisiting weighted stego-image steganalysis. In Security, Forensics, Steganography, and Watermarking of Multimedia Contents X, volume 6819 of Proc. SPIE, pages 05 1-17, 2008.

[55] A. D. Ker and T. Pevný. Batch steganography in the real world. In Proceedings of the 14th ACM Multimedia \&S Security Workshop, pages 1-10. ACM, 2012.

[56] A. D. Ker and T. Pevný. Identifying a steganographer in realistic and heterogeneous data sets. In Media Watermarking, Security, and Forensics XIV, volume 8303 of Proc. SPIE, pages 0N 1-13, 2012.

[57] A. D. Ker, T. Pevný, J. Kodovský, and J. Fridrich. The Square Root Law of steganographic capacity. In Proceedings of the 10th ACM Multimedia 8 Security Workshop, pages 107-116, 2008.

[58] Y. Kim, Z. Duric, and D. Richards. Modified matrix encoding technique for minimal distortion steganography. In Information Hiding, 8th International Workshop, volume 4437 of LNCS, pages 314-327. Springer-Verlag, 2006.

[59] Kodovský and J. Fridrich. Quantitative steganalysis using rich models. In Media Watermarking, Security, and Forensics 2013, Proc. SPIE, 2013. (to appear).

[60] J. Kodovský. Steganalysis of Digital Images Using Rich Image Representations and Ensemble Classifiers. PhD thesis, Electrical and Computer Engineering Department, 2012.

[61] J. Kodovský and J. Fridrich. On completeness of feature spaces in blind steganalysis. In Proceedings of the 10th ACM Multimedia \& Security Workshop, pages 123-132, 2008.

[62] J. Kodovský and J. Fridrich. Steganalysis in high dimensions: Fusing classifiers built on random subspaces. In Media Watermarking, Security and Forensics XIII, volume 7880, pages OL 1-13, 2011.

[63] J. Kodovský and J. Fridrich. Steganalysis of JPEG images using rich models. In Media Watermarking, Security, and Forensics 2012, volume 8303 of Proc. SPIE, pages 0A 1-13, 2012.
[64] J. Kodovský and J. Fridrich. Steganalysis in resized images. In International Conference on Acoustics, Speech, and Signal Processing. IEEE, 2013. (to appear).

[65] J. Kodovský, J. Fridrich, and V. Holub. On dangers of overtraining steganography to incomplete cover model. In Proceedings of the 13th ACM Multimedia 85 Security Workshop, pages 69-76, 2011.

[66] J. Kodovský, J. Fridrich, and V. Holub. Ensemble classifiers for steganalysis of digital media. IEEE Trans. Inf. Forensics Security, 7(2):432-444, 2012.

[67] S. Köpsell and U. Hillig. How to achieve blocking resistance for existing systems enabling anonymous web surfing. In Privacy in the Electronic Society, ACM Workshop, pages 47-58. ACM, 2004.

[68] S. Kraut and L. L. Scharf. The CFAR adaptive subspace detector is a scale-invariant GLRT. IEEE Trans. Sig. Proc., 47(9):2538-2541, 1999.

[69] S. Kullback. Information Theory and Statistics. Dover, 1968.

[70] E. Lehmann and J. Romano. Testing Statistical Hypotheses. Springer, 3rd edition, 2005.

[71] E. Li and S. Craver. A square-root law for active wardens. In Proceedings of the 13th ACM Multimedia ES Security Workshop, pages 87-92. ACM, 2011.

[72] I. Lubenko and A. D. Ker. Going from small to large data sets in steganalysis. In Media Watermarking, Security, and Forensics 2012, volume 8303 of Proc. SPIE, pages OM 1-10, 2012.

[73] S. Lyu and H. Farid. Steganalysis using higher-order image statistics. IEEE Trans. Inf. Forensics Security, 1(1):111-119, 2006.

[74] S. J. Murdoch and S. Lewis. Embedding covert channels in TCP/IP. In Information Hiding, 7th International Workshop, volume 3727 of LNCS, pages 247-261. Springer-Verlag, 2005.

[75] A. Orsdemir, O. Altun, G. Sharma, and M. Bocko. Steganalysis-aware steganography: Statistical indistinguishability despite high distortion. In Security, Forensics, Steganography, and Watermarking of Multimedia Contents X, volume 6819 of Proc. SPIE, pages 15 1-19, 2008.

[76] T. Pevný. Kernel Methods in Steganalysis. PhD thesis, Binghamton University, SUNY, 2008.

[77] T. Pevný. Detecting messages of unknown length. In Media Watermarking, Security and Forensics XIII, volume 7880 of Proc. SPIE, pages OT 1-12, 2011.

[78] T. Pevný, P. Bas, and J. Fridrich. Steganalysis by subtractive pixel adjacency matrix. IEEE Trans. Inf. Forensics Security, 5(2):215-224, 2010.

[79] T. Pevný, T. Filler, and P. Bas. Using high-dimensional image models to perform highly undetectable steganography. In Information Hiding, 12th International Conference, volume 6387 of LNCS, pages 161-177. Springer-Verlag, 2010.

[80] T. Pevny and J. Fridrich. Detection of double-compression in JPEG images for applications in steganography. IEEE Trans. Inf. Forensics Security, 3(2):247-258, 2008.

[81] T. Pevný and J. Fridrich. Multiclass detector of current steganographic methods for JPEG format. 
IEEE Trans. Inf. Forensics Security, 3(4):635-650, 2008.

[82] T. Pevný and J. Fridrich. Novelty detection in blind steganalysis. In Proceedings of the 10th ACM Multimedia \& Security Workshop, pages 167-176, 2008.

[83] T. Pevny, J. Fridrich, and A. D. Ker. From blind to quantitative steganalysis. IEEE Trans. Inf. Forensics Security, 7(2):445-454, 2012.

[84] N. Provos and P. Honeyman. Detecting steganographic content on the internet. Technical Report CITI Technical Report 01-11, University of Michigan, 2001.

[85] L. Reyzin and S. Russell. Simple stateless steganography. IACR Eprint archive, 2003. http://eprint.iacr.org/2003/093.

[86] V. Sachnev, H. J. Kim, and R. Zhang. Less detectable JPEG steganography method based on heuristic optimization and $\mathrm{BCH}$ syndrome coding. In Proceedings of the 11th ACM Multimedia \& Security Workshop, pages 131-140, 2009.

[87] U. Schmidt, Q. Gao, and S. Roth. A generative perspective on MRFs in low-level vision. In Computer Vision and Pattern Recognition, pages 1751-1758. IEEE, 2010.

[88] B. Schölkopf and A. Smola. Learning with Kernels: Support Vector Machines, Regularization, Optimization, and Beyond. MIT Press, 2001.

[89] P. Schöttle and R. Böhme. A game-theoretic approach to content-adaptive steganography. In Information Hiding, 14th International Conference, volume 7692 of $L N C S$, pages 125-141. Springer-Verlag, 2012.

[90] C. Scott and R. Nowak. A Neyman-Pearson approach to statistical learning. IEEE Trans. Inf. Theory, 51(8):3806-3819, 2005.

[91] C. E. Shannon. Coding theorems for a discrete source with a fidelity criterion. IRE Nat. Conv. Rec., 4:142-163, 1959.

[92] G. J. Simmons. The prisoner's problem and the subliminal channel. In Advances in Cryptology, CRYPTO '83, pages 51-67. Plenum Press, 1983.

[93] J. Spolsky. Joel on Software: Selected Essays. APress, 2004.

[94] J. Sun and M. F. Tappen. Learning non-local range Markov random field for image restoration. In Computer Vision and Pattern Recognition, pages 2745-2752. IEEE, 2011.

[95] T. H. Thai, F. Retraint, and R. Cogranne. Statistical model of natural images. In Proceedings IEEE, International Conference on Image Processing, ICIP 2012, pages 2525-2528. IEEE, 2012.
[96] V. N. Vapnik. Statistical learning theory. Wiley, 1998.

[97] S. Voloshynovskiy, A. Herrigel, N. Baumgaertner, and T. Pun. A stochastic approach to content adaptive digital image watermarking. In Information Hiding, 3rd International Workshop, volume 1768 of LNCS, pages 211-236. Springer-Verlag, 2000.

[98] A. B. Wagner and V. Anantharam. Information theory of covert timing channels. In Proceedings of the 2005 NATO/ASI Workshop on Network Security and Intrusion Detection, pages 292-296. IOS Press, 2008.

[99] A. Wald. Sequential tests of statistical hypotheses. Ann. Math. Stat., 16(2):117-186, 1945.

[100] C. Wang and J. Ni. An efficient JPEG steganographic scheme based on the block-entropy of DCT coefficents. In International Conference on Acoustics, Speech, and Signal Processing, pages 1785-1788. IEEE, 2012.

[101] Y. Wang and P. Moulin. Perfectly secure steganography: Capacity, error exponents, and code constructions. IEEE Trans. Inf. Theory, 55(6):2706-2722, 2008.

[102] Y. Weiss and W. T. Freeman. What makes a good model of natural images? In Computer Vision and Pattern Recognition, pages 1-8. IEEE, 2007.

[103] A. Westfeld. Steganalysis in the presence of weak cryptography and encoding. In Digital Watermarking, 5th International Workshop, volume 4283 of $L N C S$, pages 19-34. Springer-Verlag, 2006.

[104] L. Yao, X. Zi, L. Pan, and J. Li. A study of on/off timing channel based on packet delay distribution. Computers $\& 5$ Security, 28(8):785-794, 2009.

[105] S. Yuksel, J. Wilson, and P. Gader. Twenty years of mixture of experts. IEEE Trans. Neural Netw. Learn. Syst., 23(8):1177-1193, 2012.

[106] C. Zitzmann, R. Cogranne, L. Fillatre, I. Nikiforov, F. Retraint, and P. Cornu. Hidden information detection based on quantized Laplacian distribution. In International Conference on Acoustics, Speech, and Signal Processing, pages 1793-1796. IEEE, 2012.

[107] C. Zitzmann, R. Cogranne, F. Retraint, I. Nikiforov, L. Fillatre, and P. Cornu. Statistical decision methods in hidden information detection. In Information Hiding, 13th International Conference, LNCS, pages 163-177. Springer-Verlag, 2011. 